\title{
Evaluation of anti-inflammatory effect of traditional medicinal plants, Gynura segetum
}

\author{
Lay-Jing Seow*, Hooi-Kheng Beh, Amirin Sadikun, Mohd Zaini Asmawi \\ School of Pharmaceutical Sciences, Universiti Sains Malaysia, Penang, Malaysia
}

\begin{abstract}
Gynura segetum, family Asteraceae is a cultivated species and can be found growing in the tropical regions of Indonesia and Malaysia. The plant is known for its use for the treatment of cancer, inflammation, diabetes, hypertension and skin afflictions. In the current study, anti-inflammatory effect of Gynura segetum leaf has been investigated. The present study investigated the in vivo anti-inflammatory effect of Gynura segetum leaf by using hen's egg test-chorioallantoic membrane (HET-CAM) assay. Different solvent extract of Gynura segetum leaf were tested, the most active methanol extract was further fractionated and tested. Among the extracts tested, the methanol extract showed a significant good antiinflammatory effect $(76.8 \%$ inhibition at $50 \mu \mathrm{g} / \mathrm{disc})$ on the HET-CAM assay as compared with the antiinflammatory drug indomethacin $(82.1 \%$ inhibition). However, the fractionated sample exhibited a significantly lower activity in comparison to crude methanol extract. The results demonstrated that Gynura segetum leaf displayed remarkable anti-inflammatory effects, which support the folkloric uses of this plant for treatment of inflammation.
\end{abstract}

Keywords Gynura segetum; Asteraceae; HET-CAM; anti-inflammatory

\section{INTRODUCTION}

Herbal Herbal medicines are the therapeutic experiences of generations of practicing physicians of traditional medicine over hundreds of years and they are known to be oldest health care products that have been used by mankind all over the world to treat various types of ailments (Torey et al., 2010).Currently, attention is being focused on the investigation of the efficacy of traditionally used plants as they are affordable and have fewer adverse effects (Masresha et al, 2012).

Gynura segetum, family Compositae (Asteraceae) is a cultivated species and can be found growing in the tropical regions of Indonesia and Malaysia. Gynura segetum has drawn a lot of attention due to its uses in traditional medicine. The plant is known for its use for the treatment of cancer, inflammation, diabetes, hypertension and skin afflictions. A variety of chemical compounds has been identified from this genus such as flavonoids, pyrolizidine alkaloids, triterpenes, sterides and sesquiterpenoids (Zhu et al., 2013).

In previous study, the leaf extracts of Gynura segetum showed potential anti-angiogenic effects in chick embryo chorioallantoic membrane (CAM) model (Seow et al., 2011). Anti-angiogenic activity of Gynura segetum's leaf extracts provided a pharmacological basis on its folkloric use for the treatment of inflammatory diseases and cancer.

Anti-angiogenic strategies are emerging as an important tool for the treatment of cancer and inflammatory diseases.

Correspondence: Lay-Jing Seow

E-mail: layjing80@gmail.com

Received December 18, 2013; Accepted February 7, 2014; Published

February 28, 2014

doi: http://dx.doi.org/10.5667/tang.2013.0035

(C)2014 by Association of Humanitas Medicine

This is an open access article under the CC BY-NC license.

(http://creativecommons.org/licenses/by-nc/3.0/)
Therefore, researchers are growing interests in using natural anti-angiogenic agents, especially extracted from plants (Peng et al., 2009). Most of the research on anti-angiogenic agents has been directed towards inflammation treatment (Ahn et al., 2007; Jung et al., 2009; Kim et al., 2004; Krenn and Paper, 2009). Collectively, this suggested that many herbs and their phytochemicals may exert both anti-angiogenic effects and anti-inflammatory activity. Thus, there is an interest to discover local medicinal plant for anti-angiogenic and anti-inflammatory effects.

Although the plant is traditionally used in inflammatory disorders, but to the best of our knowledge, there is lack of research on the use of leaf extract of Gynura segetum in inflammation. Therefore, investigation of anti-inflammatory effects of Gynura segetum would be desirable. In the present study, the anti-inflammatory activity of Gynura segetum's leaf was evaluated in a modified version of the chicken chorioallantoic membrane assay (HET-CAM assay), as this model is an in vivo model chronic inflammation, which in this study induced by Sodium dodecyl sulfate (SDS).

\section{MATERIALS AND METHODS}

\section{Plant material}

The leaves of Gynura segetum were collected from Jabatan Pertanian Relau, Penang. A voucher specimen (No.11013) has been deposited at the herbarium of School of Biological Sciences, Universiti Sains Malaysia.

\section{Plant extraction and fractionation}

The extraction of Gynura segetum's leaves was performed similarly as described in previous study (Seow et al., 2011). Further fractionation of the most active methanol extract was carried out by resin column chromatography. The Amberlite 

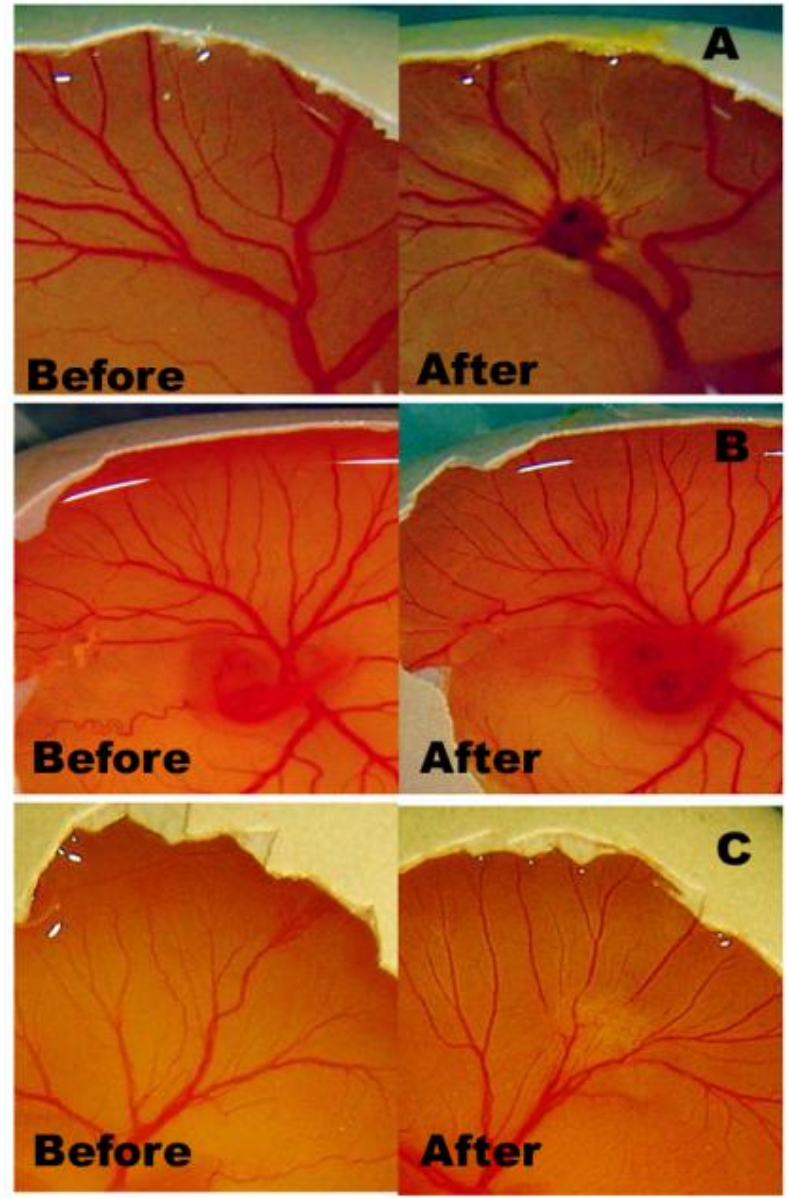

Fig. 1. HET-CAM assay. (A) Membrane irritation induced by SDS; (B) CAM treated with Indomethacin (82.1\% of inhibition); (C) The best anti-inflammatory effects of treatment with Methanol extract $(76.8 \%$ of inhibition).

XAD resins $(100 \mathrm{~g})$ were swollen in water overnight and packed into a sintered glass column measuring $25 \mathrm{~cm}$ long and $5.5 \mathrm{~cm}$ in diameter. The resins were subsequently flushed with $2 \mathrm{~L}$ of methanol and $2 \mathrm{~L}$ of water to remove impurities in the resins (Cho et al., 2009). Crude methanol extract (10 g) was

dissolved in $1 \mathrm{~mL}$ of methanol and then added in water. The sample was loaded into this reverse phase column and first eluted with water, followed by water-methanol mixture (1:1) and finally with acetone. Five fractions were collected and the solvent was removed using a rotary evaporator to afford $7.69 \mathrm{~g}$, $7.67 \mathrm{~g}, 5.22 \mathrm{~g}, 4.81 \mathrm{~g}$, and $2.18 \mathrm{~g}$ for fractions R1, R2, R3, R4, and R5 respectively. Dried sample from each fraction $(10 \mathrm{mg})$ were reconstituted in $2 \mathrm{~mL}$ of DMSO $(0.05 \%)$ and subjected to HET-CAM analysis.

\section{Preparation of test samples}

The test substances and control were dissolved in DMSO $(0.05 \%)$ to reach final concentration of $5 \mathrm{mg} / \mathrm{ml}$. For the preparation of the discs, $10 \mu 1$ of these solutions were applied dropwise on sterile filter paper of $3 \mathrm{~mm}$ diameter, dried under a laminar flow hood to give concentration of $50 \mu \mathrm{g}$ per disc. Indomethacin was applied as positive control and CAMs treated with $10 \mu \mathrm{l}$ of DMSO $(0.05 \%)$ as blank control.

\section{Hen's egg test-chorioallantoic membrane (HET-CAM) assay}

The HET-CAM assay was performed according to the method of Burgermeister et al. (2002) with some modifications. Discs of every test substances as well as positive control were placed on 10 - 15 eggs, each. The effects were compared to those of DMSO $(0.05 \%)$ discs and disc with $50 \mu \mathrm{g} /$ disc SDS. All tests run in triplicate and the evaluation was performed using a score system followed by the conversion of the score index in the proportional inhibition of inflammation: Inhibition $<40 \%$, no anti-inflammatory effect; Inhibition $40-55 \%$, uncertain antiinflammatory effect; Inhibition $55-70 \%$, weak antiinflammatory effect; Inhibition $70-85 \%$, good antiinflammatory effect; Inhibition $>85 \%$, strong antiinflammatory effect.

\section{Statistical analysis}

The data was expressed as means \pm SEM. Statistical comparison between groups was analyzed by one way analysis of variance and post hoc Tukey's test (SPSS, Inc., version 20). $p$ values less than 0.05 was considered to be significant.

\section{RESULTS AND DISCUSSION}

Table 1. Anti-inflammatory effect (HET-CAM assay) of Gynura segetum's leaf extracts, fractions and positive control

\begin{tabular}{|c|c|c|c|}
\hline \multicolumn{2}{|c|}{ Test samples } & \multirow{2}{*}{$\frac{\text { Average score }}{3.80 \pm 0.11}$} & \multirow{2}{*}{$\frac{\text { Inhibition of inflammation }(\boldsymbol{\%})}{82.12 \pm 1.68}$} \\
\hline Positive control & Indomethacin & & \\
\hline \multirow[t]{3}{*}{ Crude extracts } & $\mathrm{PE}$ & $2.05 \pm 0.23$ & $55.76 \pm 3.47$ \\
\hline & $\mathrm{CHCl}_{3}$ & $3.05 \pm 0.12$ & $70.76 \pm 1.77$ \\
\hline & $\mathrm{MeOH}$ & $3.45 \pm 0.14$ & $76.82 \pm 2.10$ \\
\hline \multirow[t]{3}{*}{ Partitions } & $\mathrm{CHCl}_{3}$ part & $2.50 \pm 0.13$ & $62.58 \pm 1.96$ \\
\hline & EA part & $2.00 \pm 0.15$ & $55.00 \pm 2.26$ \\
\hline & BU part & $2.35 \pm 0.13$ & $60.30 \pm 1.97$ \\
\hline \multirow[t]{5}{*}{ Methanolic fractions } & $\mathrm{R} 1$ & $2.10 \pm 0.12$ & $56.52 \pm 1.89$ \\
\hline & $\mathrm{R} 2$ & $1.75 \pm 0.19$ & $51.36 \pm 2.82$ \\
\hline & R3 & $1.85 \pm 0.11$ & $52.88 \pm 1.62$ \\
\hline & $\mathrm{R} 4$ & $2.20 \pm 0.13$ & $58.03 \pm 2.02$ \\
\hline & R5 & $1.90 \pm 0.07$ & $53.64 \pm 1.01$ \\
\hline
\end{tabular}

All tested samples shown significant result $(p<0.001)$ compared with control, except $\mathrm{MeOH}^{\text {a}}{ }^{\mathrm{PE}}$ : Petroleum ether extract; $\mathrm{CHCl}_{3}$ : $\mathrm{Chloroform}$ extract; $\mathrm{MeOH}$ : Methanol extract; $\mathrm{CHCl}_{3}$ part: Chloroform fraction; EA part: ethyl acetate fraction; BU part: Butanol fraction; ${ }^{\mathrm{b}}$ The result was expressed as means \pm SEM $(n=6)$. 
Anti-inflammatory activity of Gynura segetum's leaves were examined by the in vivo HET-CAM assay and the results are summarized in Table 1 . The membrane irritation induced by SDS is shown in Fig. 1A. The granuloma is strongly vascularized and a star-like capillaries network is formed surrounding the disc containing SDS. However, a complete normalized of capillaries network can be observed if antiinflammatory test substances applied together with SDS. Indomethacin as positive control exhibited strong antiinflammatory effect with inhibition $82.1 \%(50 \mu \mathrm{g} / \mathrm{disc})$. As shown in Fig. 1B, the network of blood vessels is normal and no granuloma can be observed.

Among the extract tested, the methanol extract showed good anti-inflammatory activity with inhibition $76.8 \%$ (50 $\mu \mathrm{g} / \mathrm{disc})$. The methanol extract neutralized the formation of blood vessels around the granuloma and the capillaries network appeared normal (Fig. 1C). The anti-inflammatory effect of methanol extract was almost comparable to the antiinflammatory drug indomethacin at the same concentration. Methanol extract did not show any statistically significant difference as compared with indomethacin $(p>0.05)$.

Further fractionation on the most active methanol extract was carried out and five fractions obtained were tested at the same dosage $(50 \mu \mathrm{g} / \mathrm{disc})$. All these fractions (R1 to R5) exhibited lower activity than the crude methanol extract. There was no significant difference $(p>0.05)$ between the fractions.

To the best of our knowledge, this is the first report of the anti-inflammatory activity of Gynura segetum's leaves by using chick embryo chorioallantoic membrane (CAM) assay. The major advantage of a chick embryo chorioallantoic membrane is simple to use and low cost. The CAM system allows several compounds to be tested simultaneously in the same embryo, fast and suitable for large scale screening. The vascular system of CAM is directly accessible to observation and experimentation, and there are no metabolic or hormonal influences (Mathur et al., 2006). It allows to directly studying the in vivo effect of the test compound (Min et al., 2004).

The modified of the chicken chorioallantoic membrane assay (HET-CAM assay) is an in vivo model chronic inflammation. Chronic inflammation involves proliferation, migration and recruitment of tissue and inflammatory cells, which damaged the normal tissue. Inflammatory tissue is hypoxic and induces angiogenesis through upregulation of factors such as vascular endothelial growth factor (VEGF). The angiogenic factor stimulates the proliferation and migration of endothelial cells in existing blood vessel to generate the new blood vessels. New blood vessels maintain the chronic inflammatory by transporting inflammatory cells to the inflammation site and supplying the nutrients and oxygen to the proliferating inflamed tissue (Griffioen and Molema, 2000; Jackson et al., 1997; Walsh and Pearson, 2001).

According to Jackson et al. (1997), angiogenesis and inflammation are codependent processes. An inflammatory state can promote angiogenesis, and angiogenesis can facilitate chronic inflammation. Earlier study had demonstrated that the leaf extracts of Gynura segetum reduced the growth of blood vessels and exhibited the potent anti-angiogenic effect in chick embryo chorioallantoic membrane (CAM) model (Seow et al., 2011). Preliminary phytochemical screening revealed the presence of alkaloids, terpenes, flavonoids, tannins and saponins in leaves of Gynura segetum (Seow et al., 2013). The presence of phenolic compounds in the plants indicates that this plant may have potential used in hyperglycemia, antioxidant, anticancer and anti-inflammatory etc (Doss and Phil, 2009; Rathore et al., 2012). The anti-inflammatory activity of Gynura segetum's leaves seems to be closely linked with its anti- angiogenic activity and the chemical constituents.

In the present study, the methanol extract of Gynura segetum's leaves neutralized the capillaries network and shown no granuloma tissue on CAM. These results suggested that Gynura segetum's leaves as an angiogenesis inhibitor can reduce the inflammation and help to restore the appropriate tissue and function.

\section{CONCLUSIONS}

In conclusions, this study demonstrated that the methanol extract of Gynura segetum's leaves performed potent antiinflammatory by in vivo HET-CAM assay. This finding provided a pharmacological background on the traditional use of Gynura segetum for treatment of inflammatory disease.

\section{ACKNOWLEDGEMENTS}

The authors acknowledge the financial support given by the Universiti Sains Malaysia (USM). The authors also thank the School of Pharmaceutical Sciences, USM for providing the required facilities and excellent technical assistance. The first author was supported by MyBrain 15 (MyPhD) scholarship sponsored by the Ministry of Higher Education (MOHE) Malaysia.

\section{CONFLICT OF INTEREST}

The authors have no conflicts of interest to declare.

\section{REFERENCES}

Abdillahi HS, Finnie JF, Van Staden J. Anti-inflammatory, antioxidant, anti-tyrosinase and phenolic contents of four Podocarpus species used in traditional medicine in South Africa. J Ethnopharmacol. 2011;136:496-503.

Ahn EK, Jeon HJ, Lim EJ, Jung HJ, Park EH. Antiinflammatory and anti-angiogenic activities of Gastrodia elata Blume. J Ethnopharmacol. 2007;110:476-482.

Bürgermeister J, Paper DH, Vogl H, Linhardt RJ, Franza G. LaPSvS1, a $(1 \rightarrow 3)-\beta$ - galactan sulfate and its effect on angiogenesis in vivo and in vitro. Carbohyd Res. 2002; $337: 1459-1466$.

Cho SY, Lee YN, Park HJ. Optimization of ethanol extraction and further purification of isoflavones from soybean sprout cotyledon. Food Chem. 2009;117:312-317.

Doss A, Phil M. Preliminary phytochemical screening of some Indian medicinal plants. Anc Sci Life. 2009;29:12-16.

Griffioen AW, Molema G. Angiogenesis: potentials for pharmacologic intervention in the treatment of cancer, cardiovascular diseases, and chronic inflammation. Pharmacol Rev. 2000;52:237-268.

Jackson JR, Seed MP, Kircher CH, Willoughby DA, Winkler JD. The codependemce of angiogenesis and chronic inflammation. FASEB J. 1997;11:457-465. 
Jung HJ, Song YS, Lim CJ, Park EH. Anti-inflammatory, antiangiogenic and anti-nociceptive activities of an ethanol extract of Salvia plebeia R. Brown. J Ethnopharmacol. 2009;126:355360 .

Kim SH, Song YS, Kim SK, Kim BC, Lim CJ, Park EH. Antiinflammatory and related pharmacological activities of the n$\mathrm{BuOH}$ subfraction of mushroom Phellinus linteus. J Ethnopharmacol. 2004;93:141-146.

Krenn L, Paper DH. Inhibition of angiogenesis and inflammation by an extract of red clover (Trifolim pratense L.). Phytomedicine. 2009;16:1083-1088.

Masresha B, Makonnen E, Debella A. In vivo antiinflammatory activities of Ocimum suave in mice. $\mathrm{J}$ Ethnopharmacol. 2012;142: 201-205.

Mathur R, Gupta SK, Singh N, Mathur S, Kochupillai V, Velpandian T. Evaluation of the effect of Withania somnifera root extracts on cell cycle and angiogenesis. J Ethnopharmacol. 2006;105:336-341.

Min JK, Han KY, Kim EC, Kim YM, Lee SW, Kim OH. Capsaicin inhibits in vitro and in vivo angiogenesis. Cancer Res. 2004;64:644-651.

Peng CC, Hsieh CL, Lin LY, Chyau CC, Cheng YT, Chen KC,
Peng RY. Anisotropic diffusion deviates chicken embryo chorioallantoic membrane assay (CAM) to reflect inherent therapeutic behaviors. Colloids Surf B Biointerfaces. 2009;70:29-34.

Rathore SK, Bhatt S, Dhyani S, Jain A. Preliminary phytochemical screening of medicinal plant Ziziphus mauritiana Lam. Fruits. Int J Curr Pharm Res. 2012;4:160-162.

Seow LJ, Beh HK, Abdul Majid AMS, Murugaiyah V, Ismail N, Asmawi MZ. Anti-angiogenic activity of Gynura segetum leaf extracts and its fractions. J Ethnopharmacol. 2011;134:221-227.

Seow LJ, Beh HK, Sadikun A, Asmawi MZ. Preliminary Phytochemical and Physicochemical Characterization of Gynura segetum (Lour.) Merr (Compositae) Leaf. Trop J Pharm Res. 2013;12:777-782.

Torey A, Sasidharan S, Yeng C, Yoga Latha LY. Standardization of Cassia spectabilis with respect to authenticity, assay and chemical constituent analysis. Molecules. 2010;15:3411-3420.

Walsh DA, Pearson CI. Angiogenesis in the pathogenesis of inflammatory joint and lung diseases. Arthritis Res. 2001;3:147-153.

Zhu BR, Pu SB, Wang KDG, Xu DR, Zhou HH. Chemical constituents of the aerial part of Gynura segetum. Biochem Syst Ecol. 2013;46:4-6. 